\title{
SOME REMARKS TO CONSTRUCTION OF ECR ION SOURCE HEXAPOLES ${ }^{1}$
}

\author{
J. Pivarč, J. Pivarč (Jr.) ${ }^{*}$ and M.N. El-Shazly ${ }^{* *}$ \\ Institute of Physics, Slovak Academy of Sciences, Dúbravská cesta 9, SK-842 28 Bratislava \\ Slovak Republic
}

This paper gives performance data for construction of suitable hexapoles for Electron Cyclotron Resonance Ion Sources (ECR IS). Permanent magnets are made from NdFeB magnetic material. The main attention is given to hexapoles with inner diameters of $\phi 3.6 \mathrm{~cm}$ at different hexapole thicknesses of $1.3-6 \mathrm{~cm}$. Some remarks on construction of type hexapoles are presented.

\section{INTRODUCTION}

About 30-year history of Electron Cyclotron Resonance Ion Sources (ECR IS) [1-2], which are based on the ECR has already shown that the ECR IS is an ideal tool for the production of multicharged ion states. The highly charged heavy ions are very useful not only for the ion source accelerators, but also for the investigations of the ion collision process as well as for various applications to material science.

Recently a compact ECR IS, so called "Compact $10 \mathrm{GHz}$ ECR IS" [3], composed of permanent magnet structure has been developed at University in Giessen, Germany, for atomic physics experiments. This type of ECR IS is very simple and easy for operation and maintenance without powerful electric supplies and cooling systems for getting strong magnetic field without using of coils.

By considering this, we construct ion irradiation system using ECR IS so called "NANOGUN-10B" at Bratislava.

\section{MAGNETIC FIELD CALCULATIONS OF HEXAPOLES}

The plasma in the ECR IS is kept together by a magnetic field inside magnetic bottle. The field consists of a longitudinal one, made by coils or permanent magnets and a transversal one, made by hexapole compound of permanent magnets. The magnetic bottle is the region surrounded by a closed surface of constant magnetic field $\mathrm{B}$ so that $|\mathrm{B}| \mathrm{e}=\mathrm{m}_{e} \omega_{r f}$, where $\mathrm{B}$ is the average value of the magnetic field in the region where the plasma is, e the charge of the electron, $\mathrm{m}_{e}$ the mass of

${ }^{1}$ Work supported by the VEGA L3 Ltd. firm, Záhradnícka 21, SK-821 74 Bratislava, Slovak Republic.

* Working-place address: Martin-Luther-University, Dept. of Physics, NMR Group, Friedemann-Bach-Platz 6, D-06108 Halle/Saale, Germany.

** Working-place address: Joint Institute for Nuclear Research, FLNR, 141980 Dubna, Moscow region, Russia. the electron and $\omega_{r . f}$ the microwave frequency matching the electron cyclotron frequency $\omega_{c}$. For $\omega_{c}=10 \mathrm{GHz}$ we need $|\mathrm{B}|=0.36 \mathrm{~T}$ and $|\mathrm{B}|=0.50 \mathrm{~T}$ for $\omega_{c}=14 \mathrm{GHz}$. The magnetic field inside the plasma region is lower than that on the surface of the magnetic bottle. The stronger is the magnetic field inside the magnetic bottle the higher
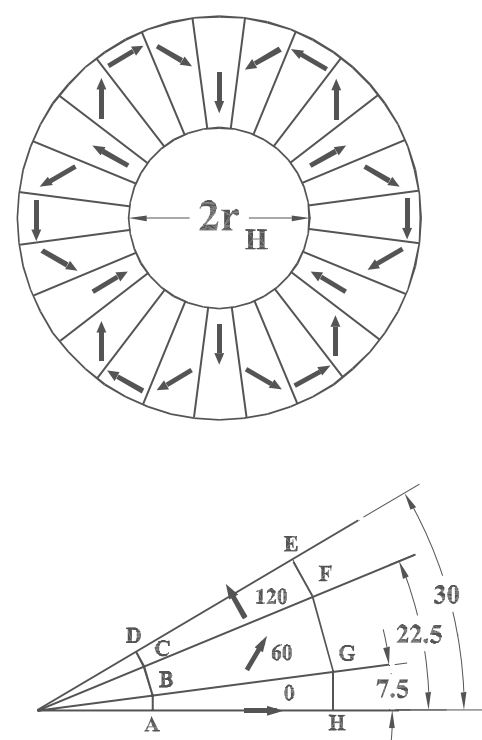

Figure 1: Cross section view of the hexapolar structures. Here, $\mathrm{r}_{H}$ is radius and $\mathrm{ABCDEFGH}$ characteristic segment of hexapole.

is the rf frequency of the resonance electrons. We thus can obtain larger plasma density that results in larger ionization possibility.

The computer program PANDIRA [4] was used at the calculations. The program calculates magnetic field on a grid in a 2-dimensional space. Permanent magnets, iron, currents and other anisotropic and isotropic materials can be defined by the user in several regions.

We have investigated 22 hexapoles with the thicknesses of $\mathrm{H}=1.4 \mathrm{~cm}$ and the inner radii of hexapoles $\mathrm{r}_{H} \in\langle 1.3$, 6) $\mathrm{cm}$. Hexapole magnets are made of $\mathrm{NdFeB}$ with a remanence of $1.1 \mathrm{~T}$ and a coercivity of $800 \mathrm{kA} / \mathrm{m}$. Each calculated hexapole consists of 24 trapezoidal segments where the angle of magnetization varies by $60^{\circ}$ from one segment to the next one. Fig. 1 shows cross section view of hexapolar structures.

A detailed description of this hexapole geometry is 
given elsewhere [5]. With this hexapole geometry

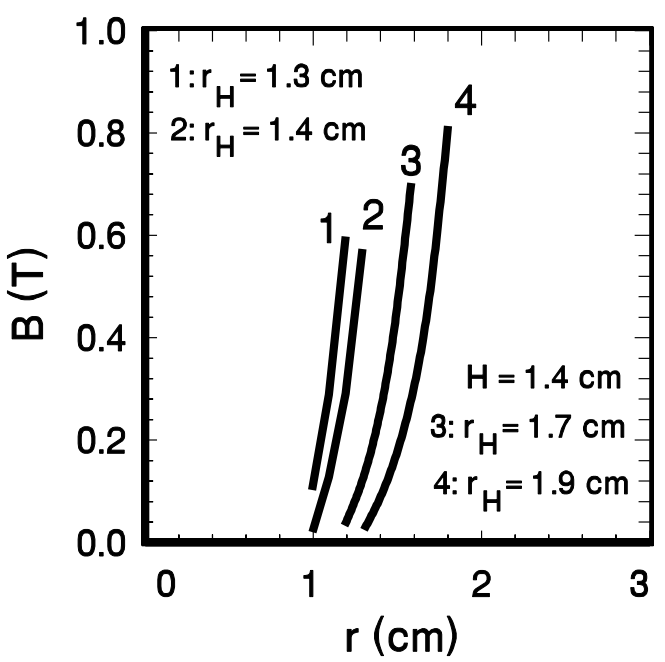

Figure 2: Magnetic field $\mathrm{B}$ inside hexapole where $\mathrm{B}_{\min }$ $=0$ for $0.9 \mathrm{~cm}<\mathrm{r}_{\min }<1.3 \mathrm{~cm}$. Here, $\mathrm{r}_{H}, \mathrm{H}$ and $\mathrm{r}$ are inner radius, thickness and cylindrical coordinate of hexapole, respectively.

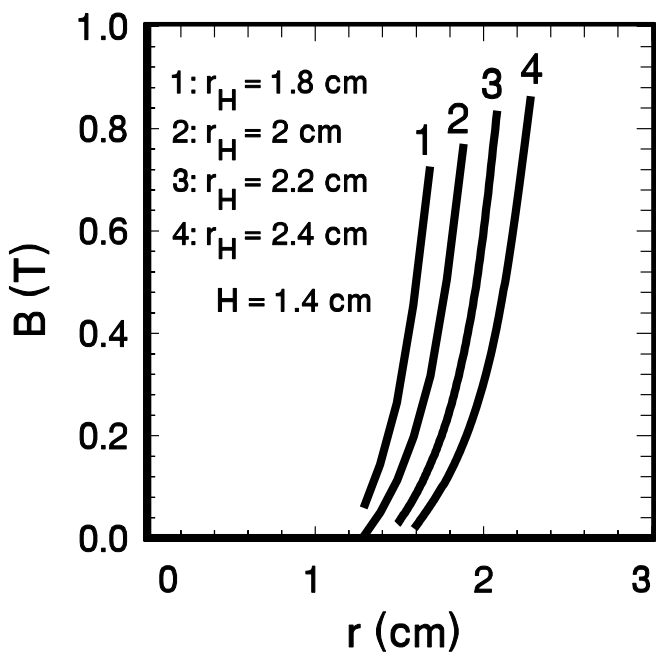

Figure 3: Magnetic field $\mathrm{B}$ inside hexapole where $\mathrm{B}_{\min }$ $=0$ for $1.1 \mathrm{~cm}<\mathrm{r}_{\min }<1.6 \mathrm{~cm}$. Here, $\mathrm{r}_{H}, \mathrm{H}$ and $\mathrm{r}$ are inner radius, thickness and cylindrical coordinate of hexapole, respectively.

a magnetic field of $1 \mathrm{~T}$ is obtained at the inner radius of hexapole $\mathrm{r}_{H}=5.2 \mathrm{~cm}$ (the thickness of hexapole $\mathrm{H}=1.4$ $\mathrm{cm})$. This value corresponds to a ratio of $\mathrm{B}_{\max } / \mathrm{B}_{E C R}=$ 2.77 at the resonance magnetic field of $\mathrm{B}_{E C R}=0.36 \mathrm{~T}$ corresponding to a cyclotron frequency of $10 \mathrm{GHz}$.

The calculations were done in the segment that is $1 / 12$ of the total hexapole in which both the mirror and the rotational symmetries are assumed. The boundary conditions were also fixed. The results of the calculations are summarized in Figs. 2 to 7. Figs. 2 to 4 show magnetic field B inside a hexapole as a function of a cylindrical coordinate $r$. These values correspond to the different cylindrical coordinates $\mathrm{r}_{\text {min }}$ at which is the magnetic field $\mathrm{B}_{\text {min }}=0$, mainly $\mathbf{r}_{\text {min }}$ $\in\langle 0.9,2.1\rangle \mathrm{cm}$. Figs. 5 to 7 show a magnetic field of B inside a hexapole as a function of a cylindrical coordinate $\mathrm{r}$. These values correspond to the different average quantities $\Delta \mathrm{r}_{a}=\sum_{i} \Delta \mathrm{r}_{i} / \mathrm{n}$ for $\Delta \mathrm{r}_{a} \in\langle 0.133,1.26\rangle \mathrm{cm}$, where $\Delta \mathrm{r}_{i}=$ $\left(\mathrm{r}_{H}-\mathrm{r}\right)_{i}, \mathrm{n}$ is the number of $\Delta \mathrm{r}_{i}, \mathrm{r}_{H}$ the inner radius of hexapole and $r$ the cylindrical coordinate. Only one value of $\Delta \mathrm{r}_{i}$ is considered for the given calculation of the magnetic field of hexapole.

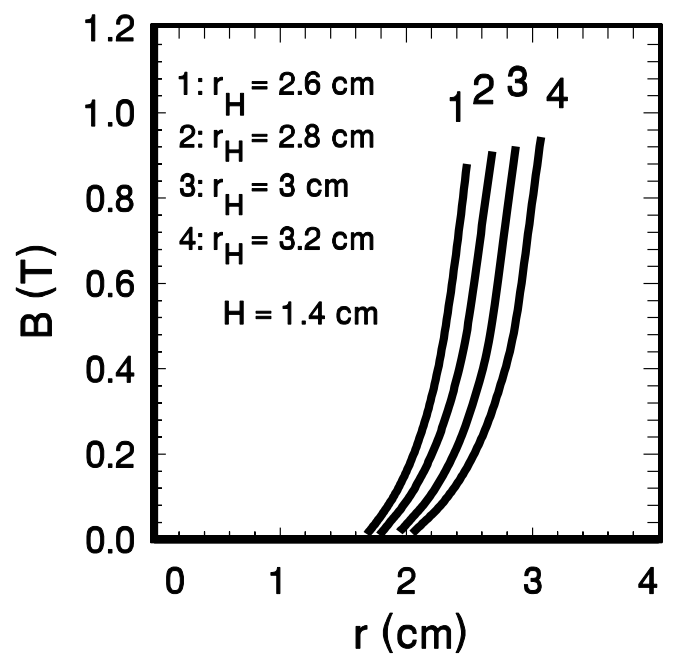

Figure 4: Magnetic field $\mathrm{B}$ inside hexapole where $\mathrm{B}_{\min }$ $=0$ for $1.6 \mathrm{~cm}<\mathrm{r}_{\min }<2.1 \mathrm{~cm}$. Here, $\mathrm{r}_{H}, \mathrm{H}$ and $\mathrm{r}$ are inner radius, thickness and cylindrical coordinate of hexapole, respectively.

\section{RESULTS}

We have chosen the thickness of hexapole $\mathrm{H}=1.4 \mathrm{~cm}$ for NANOGUN-10B to find the maximum of the magnetic field $\mathrm{B}_{\max }$ in the region of $\mathrm{r}_{H} \in\langle 1.3,6\rangle \mathrm{cm}$. Suitable radius of hexapole can be taken from the region $\mathrm{r}_{H} \in\langle 1.7$, $3.2\rangle \mathrm{cm}$ for the ECR IS NANOGUN-10B. An increasing of magnetic field at the surface of hexapole has also been found of $\Delta \mathrm{B}=0.24,0.14$, and $0.06 \mathrm{~T}$ in the regions of $\mathrm{r}_{H}$ $\in\langle 1.3,1.9\rangle \mathrm{cm}, \mathrm{r}_{H} \in\langle 1.8,2.4\rangle \mathrm{cm}$, and $\mathrm{r}_{H} \in\langle 2.6,3.2\rangle$ $\mathrm{cm}$, respectively.

To understand the influence of parameter $\Delta \mathrm{r}_{a}$ on $\mathrm{B}(\mathrm{r})$ distributions, we have shown Figs. 5 to 7 . The higher is the parameter $\Delta \mathrm{r}_{a}$ the lower is the magnetic field $\mathrm{B}$ for the given coordinate $\mathrm{r}$. A saturation of the magnetic field $\mathrm{B}_{s}$ $\left(\mathrm{B}_{s}=1.09 ; 0.8 ; 0.65\right.$, and $\left.0.55 \mathrm{~T}\right)$ at the parameters $\Delta \mathrm{r}_{a}$ $\in\langle 0.133,0.44\rangle \mathrm{cm}$ can be seen. Therefore the magnetic field at the ECR IS plasma chamber surface is $0.45 \mathrm{~T}$ for hexapole of $\mathrm{r}_{H}=1.8 \mathrm{~cm}$ and for the plasma chamber thickness of $1.77 \mathrm{~mm}$.

A well-known rule of plasma physics says that the 
higher is the mirror ratio of a magnetic trap, the smaller is the number of the particles lost from the confined plasma. The confining trap in the ECR IS is formed by the superposition of a mirror field and a hexapolar field. The higher is the hexapolar field, the higher is the confining trap and the ratios $\mathrm{B}_{\text {max }} /|\mathrm{B}|$ and $\mathrm{B}_{\max } / \mathrm{B}_{\min }$ that are also important for ECR heating.

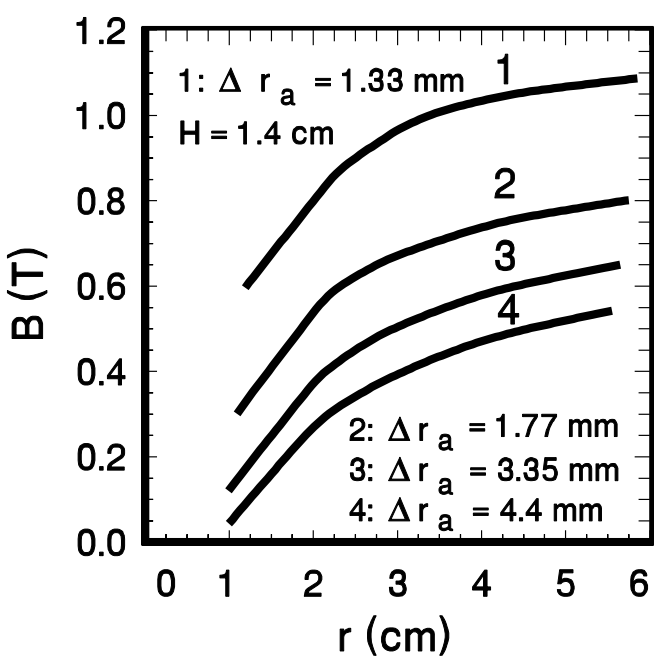

Figure 5: Magnetic field B inside hexapole where $\Delta \mathrm{r}_{a}, \mathrm{H}$ and $\mathrm{r}$ are parameter, thickness and cylindrical coordinate of hexapole, respectively.

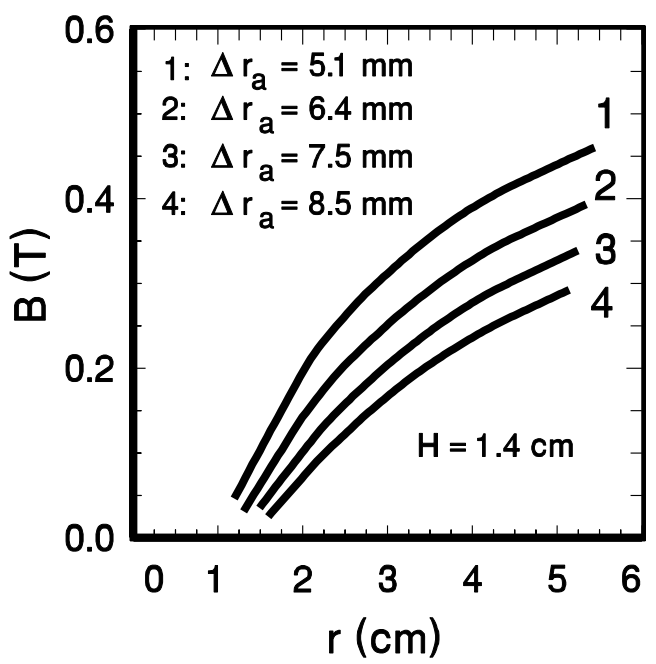

Figure 6: Magnetic field B inside hexapole where $\Delta \mathrm{r}_{a}, \mathrm{H}$ and $\mathrm{r}$ are parameter, thickness and cylindrical coordinate of hexapole, respectively.

\section{CONCLUSIONS}

The magnetic field inside hexapole with thickness of $\mathrm{H}$ $=1.4 \mathrm{~cm}$ have been calculated, as well as influence of parameters $\Delta \mathrm{r}_{a}$ on $\mathrm{B}(\mathrm{r})$. It has been shown that the higher is the parameter $\Delta \mathrm{r}_{a}$ the lower is the magnetic field $\mathrm{B}$ for the given coordinate $r$. The thickness of hexapole $\mathrm{H}=1.4 \mathrm{~cm}$ for NANOGUN-10B has been chosen to find a maximum of the magnetic field $\mathrm{B}_{\text {max }}$ in the region of $\mathrm{r}_{H} \in\langle 1.3,6\rangle$ $\mathrm{cm}$. We have shown that the best results are obtained with hexapole shape where $\mathrm{r}_{H} \in\langle 1.4,2.4\rangle \mathrm{cm}$.

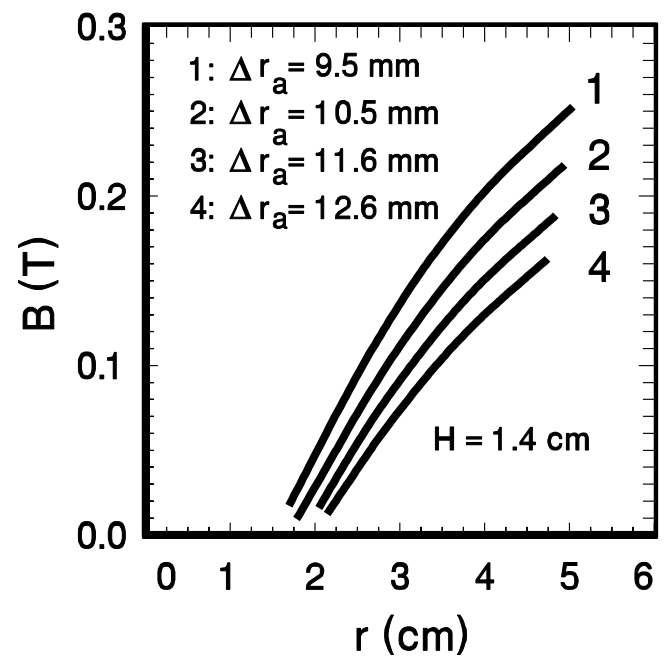

Figure 7: Magnetic field B inside hexapole where $\Delta_{r_{a}}, \mathrm{H}$ and $\mathrm{r}$ are parameter, thickness and cylindrical coordinate of hexapole, respectively.

\section{ACKNOWLEDGEMENTS}

The authors wish to thank E. Bĕták for his understanding in this work and for his help concerning the language correction of the paper. They are also indebted to P. Bandžuch and J. Zrubcová for helpful discussions. The work has been partially supported by VEGA grant No. 2/5122.

\section{REFERENCES}

[1] J. Pivarč, A.N. Lebedev, J. Pivarč (Jr.), Production of Ion Beams with the Use of ECR Ion Source, Report E1397-285, Publishing Dept., JINR Dubna, 141980 Dubna, Moscow region, Russia, 1997, p. 1-16.

[2] B. Martin, M. Grether, R. Köhrbrück, U. Stettner and H. Waldmann, The Berlin 14.5 GHz ECR IS and its Testbench, KVI Report 996, Proc. of the 11th ECR IS Workshop, Groningen, May 6-7, 1993. Ed. A.G. Drentje, University of Groningen, The Netherlands, p. 188.

[3] R. Trassl, F. Broetz, M. Pawlowsky, P. Hathiramani, M. Schlapp, J.B. Greenwood, R.W. McCullough and E. Salzborn, Status Report of a Compact $10 \mathrm{GHz}$ ECR IS, Proc. of the 13th Int. Workshop on ECRIS, 26-28 Febr., 1997, Texas A\&M University, Texas, USA, p. 143-145.

[4] POISSON/SUPERFISH Reference Manual: Report LA-UR-87126, Los Alamos, New Mexico, USA.

[5] K. Halbach, Nucl. Instr. and Meth. 169 (1980) 1. 